\title{
Editorial
}

\section{The impending threat of an Influenza pandemic}

The threat of a human influenza pandemic has greatly increased over the past several years with the emergence of highly virulent avian influenza viruses, notably H5N1 type having abilities to infect humans. This virus has been found to infect humans in several Asian, European and African countries. Previous influenza pandemics have arrived with little or no warning, but the current widespread circulation of H5N1 viruses among avian populations and their potential for increased transmission to humans and other mammalian species may afford us an opportunity to prepare for the next pandemic threat.

Three pre-requisites are considered essential for emergence of a pandemic Influenza virus: (i) appearance of a new virus subtype which can infect humans; (ii) the novel virus is able to replicate and cause serious illness in humans, and (iii) finally, the new virus is transmitted efficiently from human to human. The first two conditions have already been met by the current $\mathrm{H} 5 \mathrm{~N} 1$ avian influenza virus, the potential candidate for next pandemic, has not yet acquired the third condition for efficient human-to-human transmission. Although no one can predict with certainty when the pandemic will occur, experts warn that it is imminent and there is a great possibility that it would begin from Asia. ${ }^{1}$

The last century has witnessed three pandemics of influenza, occurring at intervals of 10 to 50 years, started without warning and spread rapidly worldwide. The great influenza pandemic of 1918-19 caused illness in more than $25 \%$ of the total population, with an estimated 40-50 million deaths within a year. Most deaths occurred in young and healthy persons in the age range of 15 to 35 years. Although the other two pandemics in 1957 and 1968 were caused by the milder viruses, each killed 1-4 million people. ${ }^{2}$

As we look towards the previous Influenza pandemics, it is found that during the 1918-19, known as Spanish flu, infecting by a H1N1 virus, caused up to 50 million deaths worldwide, mainly young and healthy adults. During the 1957-58, an Asian flu by a H2N2 subtypes, caused 1-2 million deaths worldwide, identified in China in late February, 1957. During the 1968-69, the Hong Kong flu was caused by a H3N2 that caused about 700,000 deaths and was first detected in the Hong Kong in early 1968. Each of these pandemics was preceded by development of a new virus through re-assortment of human and animal influenza virus genes. $3,4,5$

As of December 2006, outbreaks of H5N1 avian influenza viruses had occurred in domestic poultry populations in many countries in Asia, Europe and Africa. The H5N1 is an avian influenza virus subtype commonly meant for "bird flu" or "avian influenza" virus is widespread in the bird population. While H5N1 is mutating into variations which infect species not previously known to carry the virus, many of these reassortants can infect humans. A highly pathogenic variation of $\mathrm{H} 5 \mathrm{~N} 1$ is currently spreading across the world. Migrating waterfowl (wild ducks, geese, and swans) carry H5N1, often without themselves becoming sick. ${ }^{3,6}$ The H5N1 virus is gradually expanding the host range (e.g. domestic, wild and migratory birds) and spreading geographically in the affected countries as well as to other countries..$^{7,8}$

Human cases of H5N1 infections reported by WHO from 12 countries during 2006 were 112 of which total number of deaths were 79.5 Two important features have been identified: i) most cases were children and young adults, and ii) high case fatality $(62 \%)$ has been recorded. Patients developed acute respiratory distress syndrome (ARDS) with shortness of breath, viral pneumonia, acute respiratory distress on an average of 4-5 days after the first appearance of symptoms and death occurred following multi-organ failure. ${ }^{9}$

The next pandemic may cause very high morbidity and mortality in a few weeks. It is estimated that the pandemic may cause more than 1 billion cases and 2-7 million deaths. It may severely strain the health services and other essential services and cause massive social, political and economic disruption. A modest pandemic lasting over one year might cause losses as high as 3\% of Asia's and $0.5 \%$ of world's 
GDP. This is presently equivalent to about a loss of \$ 150-200 billion in GDP. Countries with pandemic preparedness and pre-existing core capacities will be able to respond quickly to pre-empt the pandemic or minimize its adverse impact. ${ }^{9}$

Three stages of typical pandemic have been identified: interpandemic period, pandemic alert period, and the pandemic period with a total of 6 phases. Inter pandemic period usually have two phases. During phase 1 , no new influenza virus subtype in humans are available but the virus may be present in animals, having low risk of human infection. During the phase 2, no new influenza virus subtype is still present in humans but poses a substantial risk of human disease. During the pandemic alert period, three phases usually occur. The 1st phase of pandemic alert period, i.e., in phase 3, human infection(s) may be present with no human-to-human spread, or at most rare instances, with spread to a close contacts. During phase 4, small clusters of human infection with limited human-to-human transmission may be present but spread is highly localized, suggesting that the virus is not well adapted to humans. During phase 5, larger cluster(s) of human infections may be present but human-to-human spread still remains localized, suggesting that the virus is becoming increasingly better adapted to humans but may not yet be fully transmissible having substantial pandemic risk. During the final stage of a typical pandemic (or phase 6), increased and sustained transmission occurs in general population. ${ }^{9}$

Responding to the crisis requires different endeavors during different phases. Pre-pandemic phase needs to (i) reduce opportunities for human infection; and (ii) strengthen the early warning system. During emergence of a pandemic virus: (iii) it should be contained or spreading should be delayed. Finally, if pandemic is declared and the virus is found spreading internationally, attempts should be centered to: (iv) reduce morbidity, mortality, and social disruption, and (v) conduct research to guide response measures.

Now, we should find out the ways how an influenza pandemic can be averted. Management should be then multisectorial. Outbreak in poultry should be managed to: (i) halt further spread in poultry population, (ii) reduce human exposure to the virus, (iii) prevention of infection among high risk persons by vaccination and provision of prophylactic antiviral drug. To reduce spreading of the virus to poultry as well as humans, construction of poultry firms in ideal conditions should be ensured, and handling of the birds should be followed by recommended and standard procedure, including selling of live poultry in open markets.

When cases of avian influenza in humans occur, there should be: (i) surveillance for animal and human infections; (ii) thorough investigation of each of the cases; (iii) aggressive management of each case with antiviral drug and isolation; (iv) antiviral for all contacts in a defined geographical area; (v) ensuring country preparedness for pandemic; and finally (vi) vaccination.

Diagnosis of avian influenza in humans can be made by viral culture, serology, rapid antigen testing, polymerase chain reaction (PCR), and immunofluorescence assays with nasopharyngeal or throat swab, nasal wash or nasal aspirate, and serum. ${ }^{8,9}$ Currently in Bangladesh, rapid antigen testing for types and PCR for few subtypes are done.

Limited evidences suggest that some antiviral drugs, notably oseltamivir (commercially known as Tamiflu, Oseflu, SKFlu Aviflu), can reduce the duration of viral replication and improve prospects of survival, provided they are administered within 48 hours following onset of symptoms. Recommendations on the optimum dose and duration of treatment for $\mathrm{H} 5 \mathrm{~N} 1$ avian influenza, also in children, need to undergo urgent review, and this is being undertaken by WHO. ${ }^{10}$ Oseltamivir is also prescribed for the poultry handers, including those involved in culling of infected birds.

In suspected cases, oseltamivir should be prescribed as soon as possible to maximize its therapeutic benefits. However, considering the significant mortality associated with $\mathrm{H} 5 \mathrm{~N} 1$ infection and evidence of prolonged viral replication, administration of the drug should also be considered in patients presenting later in the course of illness.

Md. Zafor Ullah Chowdhury Director, NIPSOM, Mohakhali, Dhaka ASM Alamgir Medical Officer, IEDCR, Mohakhali, Dhaka Saifullah Munshi Assistant Professor, Department of Virology, BSMMU, Dhaka 


\section{References}

1. US Department of Health and Human Services. HHS pandemic influenza plan. Washington, DC: US Department of Health and Human Services 2006. Web page, available at:

"http://www.hhs.gov/pandemicflu/plan/sup2.html\#app2" (accessed on $20 / 06 / 06$ )

2. Webster RG, Walker EJ. Influenza: The world is teetering on the edge of a pandemic that could kill a large fraction of the human population. Am Sc 2003; 91: 122.

3. Wikimedia Foundation Inc. H5N1. Web page retrieved from: "http://en.wikipedia.org/wiki/H5N1" [acessed on 06/03/06]

4. The Writing Committee of the WHO Consultation on Human Influenza A/H5. Avian Influenza A (H5N1) Infection in Humans. N Engl J Med 2005; 353: 1374-1385.

5. World Health Organization. Cumulative number of confirmed human cases of avian influenza $\mathrm{A} /(\mathrm{H} 5 \mathrm{~N} 1)$ reported to WHO. Geneva, Switzerland: World Health Organization; 2006. available at: http://www.who.int/csr/disease/avian_influenza/en. [accessed on 06/03/06]
6. Lee CW, Suarez DL, Tumpey TM, et al. Characterization of Highly Pathogenic H5N1 Avian Influenza A Viruses Isolated from South Korea. J Virol 2005; 79 (6): 3692-3702.

7. Kou Z, Lei FM, Yu J, et al. New genotype of avian influenza $\mathrm{H} 5 \mathrm{~N} 1$ viruses isolated from tree sparrows in China. J Virol 2005; 79 (24): 15460-15466.

8. Anonymous. Guiding principles for highly pathogenic Avian influenza surveillance and diagnostic networks in Asia. Proceedings of FAO expert meeting on surveillance and diagnosis of Avian influenza in Asia. Bankok, 21-23 July, 2004.

9. US Department of Health and Human Services. HHS pandemic influenza plan. Washington, DC: US Department of Health and Human Services 2006. Available at: "http://www.hhs.gov/pandemicflu/plan/sup2.htm1\#app2". [accessed on 12/06/06]

10. Reaney P. Chemotherapy plan on bird flu. Web page, online breaking news at: "http://www.news.com.au" (accessed on $12 / 06 / 06)$ 